Revue d'histoire de l'Amérique française

Q4. REVUE D'HISTOIRE DE L'AMÉRIQUE FRANÇAISE

\title{
L'esprit communautaire des bals des Rois au Pays des Illinois (1800-1830)
}

\section{Mikael Dumont}

Volume 73, numéro 1-2, été-automne 2019

L’Ouest franco-américain

URI : https://id.erudit.org/iderudit/1068792ar

DOI : https://doi.org/10.7202/1068792ar

Aller au sommaire du numéro

Éditeur(s)

Institut d'histoire de l’Amérique française

ISSN

0035-2357 (imprimé)

1492-1383 (numérique)

Découvrir la revue

Citer cet article

Dumont, M. (2019). L'esprit communautaire des bals des Rois au Pays des Illinois (1800-1830). Revue d'histoire de l'Amérique française, 73(1-2), 103-123. https://doi.org/10.7202/1068792ar
Résumé de l'article

Dans les trois premières décennies du XIX ${ }^{\mathrm{e}}$ siècle, les colons américains sont de plus en plus nombreux à habiter l'ancien Pays des Illinois. Malgré les changements politiques, économiques et judiciaires que cela engendre, plusieurs caractéristiques de la vie socioculturelle des habitants blancs francophones qui peuplent la région depuis le XVIII ${ }^{\mathrm{e}}$ siècle demeurent intactes, car ces derniers constituent toujours une partie importante de la population. Ainsi, leurs moeurs traditionnelles, telles que l'esprit de solidarité et de collaboration, la stabilité de la famille et la ségrégation culturelle, imprègnent grandement la sociabilité qui règne dans l'univers festif des bals de leur carnaval. 


\section{L'esprit communautaire des bals des Rois au Pays des Illinois $(\text { I800-1830 })^{\mathrm{I}}$}

Mikael Dumont

Université de Montréal

RÉSUMÉ - Dans les trois premières décennies du XIXe siècle, les colons américains sont de plus en plus nombreux à habiter l'ancien Pays des Illinois. Malgré les changements politiques, économiques et judiciaires que cela engendre, plusieurs caractéristiques de la vie socioculturelle des habitants blancs francophones qui peuplent la région depuis le XVIIIe siècle demeurent intactes, car ces derniers constituent toujours une partie importante de la population. Ainsi, leurs mœurs traditionnelles, telles que l'esprit de solidarité et de collaboration, la stabilité de la famille et la ségrégation culturelle, imprègnent grandement la sociabilité qui règne dans l'univers festif des bals de leur carnaval.

ABSTRACT - American settlers were coming in great numbers into the former Illinois Country during the first three decades of the $19^{\text {th }}$ Century. They quickly modified the political, economical and judicial systems that the local white French-speaking inhabitants were used to since the $18^{\text {th }}$ Century. However, these inhabitants were still a significant part of the population and therefore were successful in preserving several characteristics of their social life and culture. Their traditional mores, such as the spirit of solidarity and collaboration, family stability and cultural segregation, greatly permeated the sociability that reigned in the festive environment of their carnival's balls.

1. Je tiens à remercier les directeurs de ce numéro, Yves Frenette et Marie-Ève Harton, ainsi qu'Ollivier Hubert, Catherine Desbarats et les évaluateurs anonymes de la RHAF pour leurs commentaires sur les versions antérieures de l'article. 
$\mathbf{L}^{\prime}$ historiographie du Pays des Illinois a peu analysé les événements festifs. Elle se contente le plus souvent de les mentionner ou de présenter leur déroulement sans expliquer ce qui les caractérise, ni ce qu'est la nature de rôles sociaux impliqués. Par exemple, le réveillon de Noël, la réunion de famille et les étrennes du jour de l'An, les bals des Rois, la Mi-Carême, les crêpes du Mardi Gras, le mercredi des Cendres, Pâques, l'Ascension, la Pentecôte, la Nativité de Marie, la Toussaint, l'Immaculée Conception, le divertissement du dimanche, la noce et le charivari sont des réjouissances populaires qui sont simplement énumérées ${ }^{3}$. Davantage de renseignements sont fournis sur le type d'amusements qu'aiment les habitants tels que les jeux de cartes, le billard, la danse, la chasse, les combats de coqs et les courses de chevaux $x^{4}$. Toutefois, il n’est jamais précisé si ces amusements sont associés à un type de fête en particulier, ce qui aiderait pourtant à préciser quels rôles ils jouent dans la vie sociale des gens. Les quelques célébrations sur lesquelles l'historiographie élabore davantage sont la FêteDieu, la Saint-Jean-Baptiste, la Saint-Louis, la messe de minuit et l'Épiphanie, tandis que seule la guignolée est décrite avec précision5.

2. Au cours des premières décennies du XIX ${ }^{\mathrm{e}}$ siècle, le terme Pays des Illinois n’est plus utilisé pour désigner la région comprenant les villages situés de part et d'autre du fleuve Mississippi, puisque l'ensemble de ce territoire fait alors partie des États-Unis (création de l'État de l'Illinois en 1818 et de celui du Missouri en 1821). Nous emploierons tout de même ce terme dans cet article, car il s'agit d'un moyen pratique pour désigner cette région historique dans laquelle le fait français et la culture francophone sont encore présents à cette époque.

3. Joseph Médard Carrière, "Life and Customs in the French Villages of the Old Illinois Country (1763-1939)", Report of the Annual Meeting of the Canadian Historical Association, 18, 1 (1939), p. 45; Harvey Wish, "The French of Old Missouri (1804-1821): A Study in Assimilation", Mid-America: An Historical Review, 23, 3 (juillet 1941), p. 185-187; Natalia Maree Belting, Kaskaskia Under the French Regime (Urbana, University of Illinois Press, 1948), p. 68-70; Carl J. Ekberg, Colonial Ste. Genevieve: An Adventure on the Mississippi Frontier (Carbondale, Southern Illinois University Press, 2014 [1985]), p. 319-321 ; Cécile Vidal, "Les implantations françaises au pays des Illinois au XVIII ${ }^{e}$ siècle (1699-1765)", thèse de doctorat (histoire), École des Hautes Études en Sciences Sociales, Centre d’Études Nord-Américaines, 1995, p. 605 ; DeniseMarie Wilson, «Vincennes: From French Colonial Village to American Frontier Town, 1730-1820 », thèse de doctorat (histoire), West Virginia University, 1997, p. 38-39; Bonnie Stepenoff, From French Community to Missouri Town: Ste. Genevieve in the Nineteenth Century (Columbia, University of Missouri Press, 2006), p. 158, 188-190, 192-194; Margaret Kimball Brown, History as they Lived it: A Social History of Prairie du Rocher, Illinois (Carbondale, Southern Illinois University Press, 2014), p. 226-227.

4. H. Wish, "The French of Old Missouri...», p. 185-187; C. J. Ekberg, Colonial Ste. Genevieve..., p. 325328, 330-331 ; D.-M. Wilson, «Vincennes... », p. 37-39, 89-90; B. Stepenoff, From French Community to Missouri Town..., p. $187-188$.

5. J. M. Carrière, «Life and Customs...», p. 45 ; H. Wish, "The French of Old Missouri... », p. 185-187; N. M. Belting, Kaskaskia..., p. 68-70; C. J. Ekberg, Colonial Ste. Genevieve..., p. 320-322, 328-329; C. Vidal, "Les implantations françaises... », p. 605 ; B. Stepenoff, From French Community to Missouri Town..., p. 190-192; M. K. Brown, History as they Lived it..., p. 227-228, 275-278; C. Ray Brassieur, «Expressions of French Identity in the Mid-Mississippi Valley", thèse de doctorat (anthropologie), University of Missouri-Columbia, 1999, p. 109, 118-119; Fañch Postic, "Les avatars d'une quête chantée: de l'eginane à la guignolée», Port Acadie: revue interdisciplinaire en études acadiennes/Port Acadie: An Interdisciplinary Review in Acadian Studies, 13-14-15 (printemps-automne 2008, printemps 2009), p. 443-446; Anna Servaes, Franco-American Identity, Community, 
Une analyse plus poussée de certaines réjouissances populaires permettrait pourtant d'en apprendre davantage sur la nature de leurs fonctions et sur les mours de la population blanche francophone qui habite la région au cours des trois premières décennies du XIX ${ }^{e}$ siècle. Il s'agit d'une période de leur histoire qui a été peu étudiée par l'historiographie du Pays des Illinois, cette dernière s'attardant surtout sur le XVIII ${ }^{\mathrm{e}}$ siècle. Dans cet article, nous nous attarderons aux bals des Rois. Ces bals forment un cycle cohérent de festivités communautaires ${ }^{6}$ qui se suivent dans le calendrier et qui sont intimement liées les unes aux autres, car elles expriment et renforcent la solidarité entre Blancs francophones. De plus, différentes sources documentent bien ces soirées. Ce choix permet donc de brosser un tableau des plus représentatifs de la vie socioculturelle des habitants blancs francophones. L'univers festif propre à ces réjouissances populaires est caractérisé par certains traits sociaux qui sont observés au sein de cette population depuis le début de son établissement dans la région au XVIII ${ }^{e}$ siècle. Les bals des Rois sont des lieux de rencontre pour les jeunes célibataires où s'observent l'esprit de cohésion et de collaboration des habitants, l'importance de la stabilité de la famille, les inégalités socio-économiques et genrées et la ségrégation socioculturelle ${ }^{7}$ qui a cours dans cette société.

\section{PORTRAIT DE LA SOCIÉTÉ FRANCOPHONE DU PAYS DES ILLINOIS AU XVIIIE SIĖCLE}

Depuis les premiers établissements francophones au début du XVIII ${ }^{\mathrm{e}}$ siècle, la structure socio-économique qui prévaut au Pays des Illinois se révèle unique en Amérique française. Dans la région, les habitants ne possèdent pas une bande de terre étroite le long d'un cours d'eau sur laquelle ils vivent et cultivent le sol. Ils habitent plutôt des villages entourés d'un grand ensemble de terres cultivées, individuellement possédées mais ouvertes, et de pâturages communs (non clôturés) ${ }^{8}$. Ils doivent entretenir les parties de

and La Guiannée (Saint-Louis, Center for French Colonial Studies; Jackson, University Press of Mississippi, 2015).

6. Dans le présent article, les termes «communauté» et "communautaire» font référence à des ensembles de personnes de la société des Illinois qui sont unies par leur origine ethnique et leur couleur de peau.

7. Par ségrégation socioculturelle, nous entendons une ségrégation qui se manifeste lors d'événements religieux, sociaux ou familiaux tels que la messe, le repas ou encore la fête, et non lors du temps du travail.

8. Pour une comparaison des modèles de division des terres à travers les colonies françaises de l'Amérique du Nord, voir Carl J. Ekberg, French Roots in the Illinois Country: The Mississippi Frontier in Colonial Times (Urbana, University of Illinois Press, 1998), p. 5-30, 239-263. 
la clôture du grand ensemble qui se trouvent à l'avant et à l'arrière de leurs terres privées, tandis que les parties de la clôture qui longent ce grand ensemble sur les côtés sont entretenues par la collectivité. Le travail agricole repose en partie sur les efforts d'esclaves autochtones et d'origine africaine que possèdent certains cultivateurs. Bien que les esclaves représentent $43 \%$ de la population en $1752^{9}$, cette proportion diminue sensiblement au cours des décennies suivantes (1,66\% dans l'État de l'Illinois en 1820 et environ $15 \%$ dans celui du Missouri) ${ }^{10}$. Selon Cécile Vidal et Robert Morrissey, les maîtres de plus d'une dizaine d'esclaves sont rares dans la colonie française, car la majorité des cultivateurs n'en détiennent aucun ou quelques-uns. Cependant, comme la surface moyenne des terres cultivées est très grande, le travail agricole nécessite une main-d'œuvre importante que les esclaves ne peuvent combler. Les propriétaires d'esclaves doivent donc participer à la tâche et recruter des engagés blancs ${ }^{11}$. Pendant l'hiver, l'ensemble des terres servent de pâturage commun. Afin que cette ressource partagée soit utilisée efficacement tout au long de l'année, les habitants respectent certaines règles adoptées lors d'assemblées de village contrôlées par les cultivateurs les plus riches ${ }^{12}$.

La manière d'habiter le territoire et de pratiquer l'agriculture a des répercussions sur la vie socioculturelle des personnes vivant au Pays des Illinois. Les descendants d'immigrants français se côtoient et socialisent au quotidien, à la fois au village et en travaillant sur leur parcelle de terre. Selon Cécile Vidal, ce regroupement en villages permet une importante vie de relations:

9. Cécile Vidal, «Africains et Européens au Pays des Illinois durant la période française (1699-1765)», French Colonial History, 3 (2003), p. 52-55; Robert Michael Morrissey, Empire by Collaboration: Indians, Colonists, and Governments in Colonial Illinois Country (Philadelphie, University of Pennsylvania Press, 2015), p. 145-147, 156-161.

10. Le grand écart entre le nombre d'esclaves que l'on retrouve dans l'Illinois (917) et le Missouri (10 222) s'explique par le fait que l'esclavage est réglementé dans le premier État et ne l'est pas du tout dans le second. En conséquence, les nouveaux colons américains propriétaires d'esclaves s'installent presque tous au Missouri, tandis que ceux qui n'en possèdent pas optent plutôt pour l'Illinois. Pour plus de détails, voir Norman Dwight Harris, The History of Negro Servitude in Illinois, and of the Slavery Agitation in That State, 1719-1864 (Chicago, A. C. McClurg, 1904), p. 50-52; William E. Folley, The Genesis of Missouri : From Wilderness Outpost to Statehood (Columbia, University of Missouri Press, 1989), p. 253-254; John Reda, From Furs to Farms: The Transformation of the Mississippi Valley, 1762-1825 (Dekalb, Northern Illinois University Press, 2016), p. 139.

11. C. Vidal, "Africains et Européens au Pays des Illinois...», p. 54; R. M. Morrissey, Empire by Collaboration..., p. 146-147, 157-158. Le même phénomène existe en Louisiane parmi les petits propriétaires de moins de vingt esclaves. Pour plus de détails, voir Ann Patton Malone, Sweet Chariot: Slave Family and Household Structure in Nineteenth-Century Louisiana (Chapel Hill, University of North Carolina Press, 1992), p. 62 .

12. C. J. Ekberg, French Roots in the Illinois Country..., p. 31-137. 
Les maisons étaient largement ouvertes sur la rue qui était un espace public et social important. On entrait les uns chez les autres facilement, sans crier gare. Les voisins pouvaient se surveiller. Les amis venaient souper, partager un verre, jouer aux cartes ${ }^{13}$.

Les rapports sociaux quotidiens des habitants au travail et dans la gestion des terres favorisent le développement de la coopération, de la solidarité et d'un puissant esprit communautaire. Selon Morrissey, ces caractéristiques permettent aux communautés blanches francophones de maintenir, malgré les différentes autorités françaises, espagnoles et américaines qui se succèdent dans la seconde moitié du XVIII ${ }^{e}$ siècle, une culture locale fondée sur la stabilité de la famille et l'agriculture. Ces deux historien(ne)s précisent aussi que tous ne font pas partie de ces communautés stables et solidaires. Les riches propriétaires au sommet de l'échelle sociale, d'une part, et les engagés et les voyageurs de la traite des fourrures au bas de cette même échelle d'autre part, ne partagent pas nécessairement le même sentiment de solidarité avec la majorité des cultivateurs. De plus, les esclaves possédés par les Blancs sont encore plus en marge de la société. Vidal et Morrissey nuancent donc les propos de leur collègue Carl J. Ekberg. Selon ce dernier, la gestion commune des terres aux Illinois est unique en Amérique du Nord, car elle s'oppose à l'agriculture individualiste pratiquée dans les autres régions. Il prétend que ce modèle reproduit un "système" paysan et une "mentalité» paysanne semblables à ce que l'on retrouve dans le nord de la France, qui mettent l'accent sur l'interdépendance entre les habitants et qui assurent la cohésion et la stabilité de toute la société des Illinois. Cependant, en réalité, le Pays des Illinois, malgré la présence de petites communautés blanches cohésives, est une société très fortement hiérarchisé $e^{14}$.

Les esclaves, majoritairement d'origine africaine, partagent l'espace du travail avec plusieurs Blancs, sans être sur un pied d'égalité. Comme dans les autres systèmes esclavagistes de cette époque, les maîtres leur infligent souvent des punitions corporelles. Toutefois, toujours selon Vidal et Morrissey, puisque l'agriculture est fondée sur des cultures de type européen (conditions de travail moins pénibles que sur de grandes plantations), que

13. C. Vidal, «Les implantations françaises...», p. 610.

14. C. J. Ekberg, Colonial Ste. Genevieve ..., p. 458-460 ; C. J. Ekberg, French Roots in the Illinois Country..., p. 1-3, 239-263 ; C. Vidal, "Les implantations françaises...», p. 19-20, 560-579; C. Vidal, "Africains et Européens au Pays des Illinois... », p. 58-60; Robert Michael Morrissey, «Bottomlands and Borderlands: Empires and Identities in the Illinois Country, 1673-1785 ", thèse de doctorat (histoire), Université Yale, 2006, p. 250-290. 


\section{Figure I}

\section{Une habitation au Pays des Illinois}

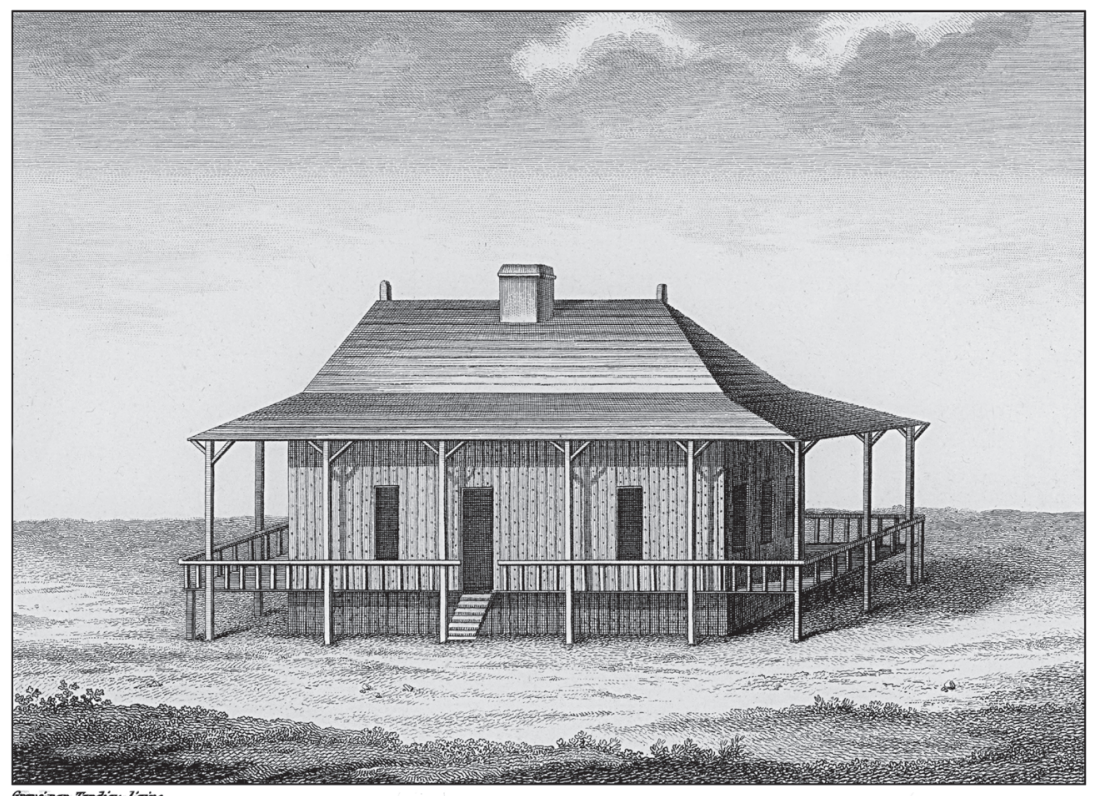

Trench Habitation in the Cointry of the Mllinois.

Tardieu l'ainé, A Journey in North America, Containing a Survey of the Countries Watered by the Mississipi, Ohio, and Missouri..., Paris, Arthur Bertrand, 1826.

l'ajout de nouveaux esclaves est marginal après les années 1730 et que ces derniers peuvent facilement s'enfuir dans un territoire isolé, ils sont généralement mieux traités que dans les autres régions esclavagistes de l'Amérique française (Louisiane et Antilles). Cet état de fait explique pourquoi les esclaves sont intégrés dans certains aspects de la vie villageoise des Blancs. Par exemple, ils sont christianisés, car ils sont baptisés et se marient à l'église paroissiale ${ }^{15}$. Cette intégration partielle ne signifie pas pour autant que les esclaves noirs font partie de la vie socioculturelle des Blancs ${ }^{16}$, car aux yeux de ces derniers, la couleur de leur peau indique qu'ils sont des êtres infé-

15. C. Vidal, «Les implantations françaises...», p. 19-20; C. Vidal, "Africains et Européens au Pays des Illinois... », p. 54-56, 59-65 ; R. M. Morrissey, Empire by Collaboration..., p. 145-147, 156-161.

16. C. Vidal, "Africains et Européens au Pays des Illinois...», p. 55; R. M. Morrissey, Empire by Collaboration..., p. 158. 
rieurs, de sorte qu'une barrière doit toujours exister entre les deux groupes ${ }^{17}$. De leur côté, les nations autochtones alliées qui peuplent la région ne vivent pas dans les villages des Blancs. Bien qu'ils soient nombreux à les côtoyer lors de la chasse et des campagnes militaires par exemple, les Autochtones vivent dans des villages séparés des villages français ${ }^{18}$. Finalement, il est vrai que les femmes blanches possèdent certains droits issus de la Coutume de Paris, telle une portion égale de l'héritage familial, et que les plus âgées, épouses ou veuves, s'occupent parfois des transactions financières de leur famille. Cependant, la puissance culturelle du patriarcat reste très forte. La plupart des femmes ne jouent généralement aucun rôle socio-économique et doivent surtout s'occuper de l'éducation des enfants et des tâches domestiques. Elles sont toujours en position de dépendance à l'égard des hommes qui détiennent le réel pouvoir sur les plans économique, politique et juridique ${ }^{19}$.

Le portrait décrit ci-dessus prévaut presque partout jusqu'à la fin du XVIII ${ }^{\mathrm{e}}$ siècle. En plus d'être à l'origine des caractéristiques des bals des Rois qui seront décrits plus loin, il permet à la vie socioculturelle des francophones de subir en général peu de changements. Il existe cependant une exception, car les habitants demeurant de part et d'autre du Mississippi vivent deux contextes sociopolitiques différents. Depuis le traité de Paris de 1763, la rive ouest du fleuve est sous juridiction espagnole et fait partie de la colonie louisianaise, tandis que la rive est, d'abord propriété des Britanniques, appartient désormais aux Américains depuis le traité de Versailles de 1783. Dès les années 1760, des soldats britanniques puis, rapidement dans les années 1780 , des soldats et des colons américains arrivent dans le village de Kaskaskia, situé sur la rive orientale. Ils chamboulent l'ordre établi en réquisitionnant les biens et la nourriture des habitants francophones et en les empêchant de pratiquer certaines de leurs coutumes religieuses. De nombreux francophones décident alors de déménager sur la rive ouest à Sainte-Geneviève en deux vagues distinctes (années 1760 et fin des années 1780). Kaskaskia représente un cas unique, car dans les autres

17. Pour plus de détails sur le concept biologique de la race dans le monde atlantique français au XVIII siècle, notamment au Pays des Illinois ainsi qu'en Louisiane, voir Guillaume Aubert, " "The Blood of France": Race and Purity of Blood in the French Atlantic World», The William and Mary Quarterly, 61, 3 (juillet 2004), p. 468-478; C. Vidal, "Africains et Européens au Pays des Illinois...», p. 61-63; Cécile Vidal, "Caribbean Louisiana: Church, Métissage, and the Language of Race in the Mississippi Colony during the French Period", dans Cécile Vidal, dir., Louisiana: Crossroads of the Atlantic World (Philadelphie, University of Pennsylvania Press, 2014), p. 125-146.

18. R. M. Morrissey, Empire by Collaboration..., p. 127-130.

19. W. E. Folley, The Genesis of Missouri..., p. 108-111; Susan C. Boyle, «Did She Generally Decide? Women in Ste. Genevieve, 1750-1805 ", The William and Mary Quarterly, 44, 4 (octobre 1987), p. 775-789. 
villages situés à l'est du Mississippi, c'est-à-dire Cahokia, Prairie-du-Rocher et Vincennes, les francophones réussissent à cohabiter avec les anglophones. Sur la rive occidentale du fleuve, les premiers colons américains n'arrivent qu'à la fin des années 1790 et ne s'implantent véritablement en grand nombre qu'après l'achat de la Louisiane par les États-Unis en $1803^{20}$.

Malgré une présence américaine de plus en plus forte, il faut attendre le second quart du XIX ${ }^{\mathrm{e}}$ siècle avant qu'elle n'influence et ne modifie véritablement le mode de vie des francophones blancs. En particulier, le modèle des terres cultivées à aire ouverte et des pâturages communs disparaît progressivement sur les deux rives du Mississippi, en raison de l'influence des lois et des pratiques américaines et de l'augmentation de la population. Les colons américains chassent aussi rapidement les nations autochtones de la région. De plus, la langue anglaise s'impose graduellement dans la sphère publique. Malgré ces changements, les communautés d'habitants blancs francophones constituent toujours une partie importante de la population. En raison de la persistance des réseaux francophones, ce que Robert Englebert nomme le monde fluvial français, plusieurs Canadiens en provenance de Montréal s'établissent également aux Illinois entre la fin du XVIII ${ }^{e}$ et le début du XIX ${ }^{e}$ siècle. Entre 1755 et 1805, 34\% des mariages célébrés dans la région comprennent un époux canadien ${ }^{21}$. Le français demeure donc la langue d'usage lorsque les habitants parlent entre eux ou à leurs esclaves, qu'ils vont à confesse ou qu'ils assistent à la messe dans l'église paroissiale. L'historiographie retient de ce fait que leur vie socioculturelle est peu altérée jusqu'aux années 1830-184022. Toutefois, en comparaison avec l'analyse détaillée effectuée pour le XVIII ${ }^{e}$ siècle, ces quelques éléments ne permettent pas de déterminer si les mœurs de cette population restent intactes dans les premières décennies du XIX ${ }^{e}$ siècle. L'analyse des bals des Rois démontrera que cela se révèle être le cas.

20. C. J. Ekberg, Colonial Ste. Genevieve..., p. 328, 397-399, 427-430; D.-M. Wilson, "Vincennes...», p. 42-43, 343-344, 348, 354-355, 363-364 ; M. K. Brown, History as they Lived it..., p. 212-213, 224 ; R. M. Morrissey, Empire by Collaboration..., p. 232-233 ; John Reda, From Furs to Farms..., p. 24-28; Tangi Villerbu, «Pouvoir, religion et société en des temps indécis: Vincennes, 1763-1795», Revue d'histoire de l'Amérique française, 62, 2 (automne 2008), p. 203, 211-213.

21. Robert Englebert, «Beyond Borders: Mental Mapping and the French River World in North America, 1763-1805", thèse de doctorat (histoire), Université d’Ottawa, 2010, p. 58.

22. H. Wish, "The French of Old Missouri...", p. 173, 188; C. J. Ekberg, Colonial Ste. Genevieve..., p. 92-94, 103, 123-124, 462-468; C. J. Ekberg, French Roots in the Illinois Country..., p. 240-255 ; B. Stepenoff, From French Community to Missouri Town..., p. 19-21, 66, 187; M. K. Brown, History as they Lived it..., p. 205, 214; Carl J. Ekberg, François Vallé and his World: Upper Louisiana before Lewis and Clark (Columbia, Missouri, University of Missouri Press, 2002), p. 293. 


\section{DES BALS DE CARNAVAL AXÉS SUR LA RENCONTRE}

La saison du carnaval au Pays des Illinois est associée à l'organisation de nombreux bals qui sont interconnectés et qui portent un nom spécifique, c'est-à-dire les bals des Rois. Dans les villages de la région, la saison débute à la fête de l'Épiphanie lorsque la majorité de la population blanche francophone, peu importe le rang social de chacun, a pour coutume de se rassembler afin de partager un grand repas festif préparé à partir des denrées récoltées lors de la quête de la guignolée du 31 décembre. Au cours de la soirée, on sert un gâteau contenant quatre fèves qui permettent de couronner quatre filles ou quatre garçons qui désignent ensuite à leur tour quatre rois ou quatre reines. Les gens présents décident ensuite de l'emplacement du premier bal des Rois de la saison du carnaval. Les quatre rois sont chargés de l'organiser et d'en payer les coûts. À la fin de ce premier bal, les quatre reines choisissent, en les embrassant, quatre nouveaux rois et ces derniers sélectionnent eux aussi quatre nouvelles reines. Ces quatre nouveaux rois, à l'instar des quatre premiers, doivent organiser et financer le prochain bal des Rois. Ainsi, lors de chacun de ces bals, quatre nouvelles filles et quatre nouveaux garçons deviennent reines et rois. Ces événements festifs se succèdent jusqu’à la fin du carnaval, c'est-à-dire au début du Carême lors du mercredi des Cendres. Chaque village comprend sa série de bals qui se déroulent une ou quelques fois par semaine. Ces réjouissances populaires ont suscité très peu d'intérêt dans l'historiographie ${ }^{23}$. Pourtant, elles forment un cycle cohérent de festivités communautaires qui expriment et renforcent la solidarité entre Blancs francophones. Ce cycle est structuré par le système de renouvellement des rois et des reines dont l'objectif est de former les futurs couples et d'assurer par le fait même la stabilité des familles de cultivateurs.

De nombreuses sources décrivent en détail les bals des Rois qui se déroulent à Sainte-Geneviève, à Kaskaskia, à Prairie-du-Rocher, à Cahokia, à Carondelet, à Saint-Louis, à Saint-Charles, à Florissant ainsi qu’à Vincennes dans les trois premières décennies du XIX ${ }^{\mathrm{e}}$ siècle. Plusieurs d'entre elles sont rédigées par des auteurs américains qui possèdent une vision très stéréotypée des habitants blancs francophones du Pays des Illinois. Selon Edward Watts, ces auteurs utilisent les descendants des immigrants français «to tell a different American story, not in the hope of re-creating the French frontier in itself - that was a lost cause - but rather to remind American readers of

23. H. Wish, "The French of Old Missouri...», p. 186; N. M. Belting, Kaskaskia..., p. 68-70; B. Stepenoff, From French Community to Missouri Town..., p. 192. 
a set of values the French had embodied and the Anglos had displaced ${ }^{24}$ ». En d'autres mots, ils véhiculent que ces francophones proposent «an alternative whiteness and an alternative American identity. Such writers held up the French settlements as less materialistic, less racialized, more democratic, more exciting, and ultimately freer than the Anglo-American civilization ${ }^{25}$.» Cette opinion subjective teinte la description de plusieurs auteurs sur les bals des Rois au Pays des Illinois. Il s'agit du cas, par exemple, des récits d'Henry Marie Brackenridge, de John Reynolds et de James Hall ${ }^{26}$. L'avocat Brackenridge passe une partie de son enfance à Sainte-Geneviève (17931796) après y avoir été envoyé par son père pour apprendre le français. Dans son ouvrage le plus connu, Views of Louisiana, il relate que les bals des Rois regroupent les gens du village dans une atmosphère conviviale où les différences de statut social sont peu visibles:

There was scarcely any distinction of classes in the society. The wealthy or more intelligent, would of course be considered as more important personages, but there was no difference clearly marked. They all associated, dressed alike, and frequented the same ball room. [...] The number of persons excluded was exceedingly small ${ }^{27}$.

De son côté, John Reynolds, juge et gouverneur de l'Illinois, passe son adolescence à Kaskaskia entre 1800 et 1807 et ouvre son premier cabinet d'avocat à Cahokia en 1814. Dans son livre sur l'histoire de l'Illinois qu'il publie en 1852, il affirme que les bals des Rois, qu'il a observés dans ces deux villages au début du XIX ${ }^{\mathrm{e}}$ siècle, comportent une effervescence et une animation qui entraînent les plus âgés, les plus jeunes, les riches et les pauvres, comme s'ils formaient une seule famille. En fait, «all meet together in good feeling and mingle together with hearts overflowing with the ecstasies of

24. Edward Watts, In This Remote Country: French Colonial Culture in the Anglo-American Imagination, 1780-1860 (Chapel Hill, University of North Carolina Press, 2006), p. 5.

25. E. Watts, In This Remote Country..., p. 13.

26. Pour plus de détails sur ces trois auteurs et sur leurs récits, voir Fred Kniffen, «Reviewed Work: Views of Louisiana, Together with a Journal of a Voyage up the Missouri River, in 1811 by Henry Marie Brackenridge ", Louisiana History: The Journal of the Louisiana Historical Association, 4, 1 (hiver 1963), p. 97-98; Anonyme, "Brackenridge, Henry Marie, (1786-1871)», Biographical Directory of the United States Congress, $<$ http:/ / bioguide.congress.gov/scripts/biodisplay.pl?index=B000732>; Anonyme, "John Reynolds", dans Portrait and Biographical Album of Champaign County, Illinois, Containing Full Page Portraits and Biographical Sketches of Prominent and Representative Citizens of the County, Together with Portraits and Biographies of all the Governors of Illinois, and of the Presidents of the United States (Chicago, Chapman Brothers, 1887), p. 123-124; Anonyme, "Hall, Hon. James", dans The Biographical Encyclopaedia of Ohio of the Nineteenth Century (Cincinnati et Philadelphie, Galaxy Publishing Company, 1876), p. 660-661.

27. Henry Marie Brackenridge, Views of Louisiana; Together with a Journal of a Voyage up the Missouri River, in 1811 (Pittsburgh, Cramer, Spear and Richbaum, 1814), p. 135. 
merriment ${ }^{28}{ }^{\prime}$. Quant à lui, James Hall est procureur général, puis juge du circuit de l'Illinois au cours des années 1820 et 1830, ce qui l'amène à se promener à travers l'État pour y administrer la justice. Il côtoie alors fréquemment les francophones de Kaskaskia, de Cahokia et de Prairie-duRocher. Ces rencontres inspirent Hall qui publie deux nouvelles dont l'intrigue se passe dans un village français de la région. Dans sa première nouvelle, qui se déroule au début du XIX ${ }^{\mathrm{e}}$ siècle, le narrateur raconte que les bals rassemblent toutes les personnes blanches du village, jeunes et vieux ${ }^{29}$. Dans sa seconde nouvelle, dont l'action a lieu à la fin du XVIII ${ }^{e}$ siècle, le narrateur précise que les communautés blanches francophones des Illinois forment une seule grande famille où règnent l'harmonie et l'unité, car bien que «some were poorer than others, they all mingled in the same dance; and as none claimed superiority, or attempted to put others to shame by affecting a show of wealth, there was little envy or malice ${ }^{30}$ ».

Ces trois auteurs racontent que la presque totalité des habitants blancs francophones partage l'espace de la fête, car plusieurs niveaux de fortune différents y sont représentés. Ils laissent entendre que ce mélange de personnes s'apparente à un estompement de la hiérarchie des rapports sociaux. Leurs propos suggèrent qu'il règne dans les bals une ambiance égalitaire qui élimine les conventions habituelles et qui rassemble les habitants formant ainsi un clan. Toutefois, tel que l'explique Edward Watts, ces trois hommes font partie d'un groupe d'auteurs américains qui, dans leur vie publique, ont toujours valorisé la défense et le maintien de la diversité dans la nation américaine. Cela explique pourquoi, dans leurs écrits, ils décrivent toujours les Blancs d'origine française des Illinois en opposition aux Américains anglophones. Ils voient en ces habitants des gens moins matérialistes et plus gentils, égalitaires et pacifiques. Ils regrettent de voir que, «by sheer bulk and energy, the French islands of bucolic peace will be lost in the turbulent sea of American immersion ${ }^{31}$ ». Ils voudraient voir ces mœurs françaises se perpétuer, car sinon ce serait «a step away from wisdom and happiness, albeit a step toward progress and profit $^{32}$ ». Leur volonté de promouvoir la singularité française explique

28. John Reynolds, The Pioneer History of Illinois Containing the Discovery in 1673, and the History of the Country to the Year 1818, When the State Government Was Organized ( $2^{\mathrm{e}}$ édition, Chicago, Fergus Printing Company, 1887), p. 71-72.

29. James Hall, "The French Village», dans Tales of the Border (Cincinnati, Truman, 1835), p. 119.

30. James Hall, "A Legend of Carondelet; or, Fifty Years Ago », dans Legends of the West (New York, T. L. Magagnos and Company, 1854), p. 330.

31. E. Watts, In This Remote Country..., p. 82.

32. E. Watts, In This Remote Country..., p. 83. 
pourquoi ils insistent constamment sur les caractéristiques stéréotypées de ces habitants ${ }^{33}$.

La prise en compte de leur subjectivité et l'analyse méticuleuse de leurs descriptions, ainsi que de celle d'autres auteurs, permettent de croire que l'égalité dans les bals des Rois se manifeste davantage en apparence qu'en réalité. L'égalité est un des traits sociaux stéréotypés qu'ils associent aux Blancs francophones des Illinois et leurs récits ont par conséquent tendance à survaloriser cet aspect. Dans le même temps, une lecture attentive permet d'observer que l'égalité magnifiée dans les récits est dans les faits plutôt secondaire et superficielle. Elle se définit surtout par l'uniformité des vêtements et par des contacts déférents entre des personnes de divers statuts sociaux. En d'autres mots, ces auteurs relatent fidèlement les bals qu'ils observent, mais amplifient l'importance qu'y occupe la notion d'égalité. En effet, le regroupement de la majorité des personnes blanches francophones dans un même lieu, où la plupart s'habillent uniformément et entretiennent de bons rapports, s'explique par la force de l'esprit communautaire sans qu'on puisse en inférer la manifestation d'une véritable égalité sur le plan sociographique. En ce début du XIXe siècle, ils ont simplement préservé l'habitude de se côtoyer, de socialiser et de collaborer, habitude qu'ils ont développée dans les décennies antérieures en gérant ensemble certains aspects publics de leur vie villageoise, tels que le grand ensemble de terres ouvertes. Le cadre social des bals des Rois, bien qu'il produise une égalité symbolique, met surtout en lumière les traits sociaux caractéristiques de la société fortement hiérarchisée du Pays des Illinois. L'étude des sources permettra aussi d'observer que l'élément le plus important des festivités est le déroulement du bal à proprement parler avec ses nombreuses danses et son processus de couronnement. Ce cadre festif fait partie des occasions formalisées, régulées par la communauté, de rencontre entre les jeunes célibataires blancs francophones. Ces soirées favorisent le contrôle sur la formation des couples ${ }^{34}$. Les règles de la sociabilité au sein de cette population pourront donc être mises en lumière en analysant le comportement des personnes présentes lors de ces soirées.

Tout d'abord, le processus de renouvellement des quatre rois et des quatre reines constitue un excellent exemple pour exposer l'égalité sym-

33. E. Watts, In This Remote Country..., p. 47-50, 79-89, 205-217.

34. Pour plus de détails sur les rôles des bals dans les milieux ruraux au XIX ${ }^{\mathrm{e}}$ siècle, voir Pierre Bourdieu, "Célibat et condition paysanne», Études rurales, 5-6 (1962), p. 86-108; Ellen K. Rothman, Hands and Hearts: A History of Courtship in America (New York, Basic Books Inc., 1984), p. 24; Peter Ward, Courtship, Love, and Marriage in Nineteenth-Century English Canada (Montréal et Kingston, McGill-Queen's University Press, 1990), p. 66-69. 
bolique des bals. Ce sont toujours les quatre reines qui, à la fin de chaque bal, sélectionnent chacune un garçon pour en faire le roi du prochain bal. Lorsqu'elles ont fait leur choix, elles avancent vers lui, lui remettent un bouquet de fleurs qu'elles épinglent sur leur habit et l'embrassent. Ensuite, les quatre nouveaux rois font de même pour choisir chacun une nouvelle reine pour le prochain bal. Par exemple, dans la première nouvelle de James Hall, le narrateur, un étranger américain qui arrive dans le village lors du premier jour du carnaval, prend part au bal des Rois qui a lieu chez l'un des habitants et se trouve à être l'un des nouveaux rois choisis par l'une des reines:

By and by the dancing ceased, and four young ladies of exquisite beauty, who had appeared during the evening to assume more consequence than the others, stood alone on the floor. For a moment their arch glances wandered over the company who stood silently around, when one of them, advancing to a young gentleman, led him into the circle, and taking a large bouquet from her own bosom, pinned it upon the left breast of his coat, and pronounced him "KING!». The gentleman kissed his fair elector, and led her to a seat. Two others were selected almost at the same moment. The fourth lady hesitated for an instant, then advancing to the spot where I stood, presented me her hand, led me forward, and placed the symbol on my breast, before I could recover from the surprise into which the incident had thrown me. I regained my presence of mind, however, in time to salute my lovely consort; and never did king enjoy, with more delight, the first fruits of his elevation for the beautiful Gabrielle, with whom I had just danced, and who had so unexpectedly raised me, as it were, to the purple, was the freshest and fairest flower in this gay assemblage ${ }^{35}$.

Le portrait esquissé dans cette nouvelle de James Hall est sensiblement le même dans trois autres sources ${ }^{36}$, dont le journal de voyage du capitaine écossais des Royal Engineers, Donald MacDonald. Ce dernier accompagne le socialiste utopique gallois Robert Owen aux États-Unis afin d'y dénicher un endroit pour établir une communauté socialiste ${ }^{37}$. De passage dans le village de Vincennes en janvier 1825, MacDonald rapporte les dires de Robert Morgan Evans, un Américain originaire du Kentucky qui habite dans l'Indiana depuis 1805 et qui a déjà vécu à Vincennes entre 1809 et

35. J. Hall, «The French Village», p. 117-118.

36. Le processus de renouvellement des quatre rois et des quatre reines est également décrit par John Reynolds et Christian Schultz. Pour plus de détails, voir J. Reynolds, The Pioneer History of Illinois..., p. 72; Christian Schultz, Travels on an Inland Voyage through the States of New-York, Pennsylvania, Virginia, Ohio, Kentucky and Tennessee, and through the Territories of Indiana, Louisiana, Mississippi and New-Orleans; Performed in the Years 1807 and 1808; Including a Tour of Nearly Six Thousand Miles (New York, Isaac Riley, 1810), vol. 2, p. 61.

37. Pour plus de détails sur le projet de Robert Owen, voir Douglas F. Dowd, «Robert Owen», Encyclopaedia Britannica, <https://www.britannica.com/biography/Robert-Owen>. 
$1811^{38}$, à propos de sa participation au processus de couronnement dans les bals des Rois des francophones du village ${ }^{39}$ :

The first ball he was at he was made acquainted with one of their customs by having the trick played upon himself. While standing in conversation with a gentleman [ $\left.{ }^{\star}\right]$ at one end of the ball-room, a lady came up entered into conversation with him \& before he was aware of it sewed a ticket on the lappet of his coat. This was a signal that he was engaged to give the next ball $\&$ be the king of it. His friend then informed him that he must choose his queen from among the ladies in the room. ${ }^{x}\left[{ }^{x}\right.$ in the same manner, by fastening the ticket, when unobserved, on the sleeve of one of them, then] presenting his hand to her, kissing her cheek (not her mouth which would be an offence) and dancing with her ${ }^{40}$.

Dans leur description respective, Hall et MacDonald montrent comment les filles prennent leur temps, pendant que la salle est silencieuse, pour aller vers le garçon qu'elles préfèrent. Elles disposent d'une certaine liberté dans leur choix puisque, comme ils le révèlent, elles n'hésitent même pas à prendre pour roi des étrangers anglophones. Le couronnement constitue un rituel de courtoisie qui laisse aux filles le choix du partenaire qu'elles désirent fréquenter ${ }^{41}$. La succession de choix des filles et des garçons offre plus qu'une option de fréquentation à ces jeunes célibataires. Ainsi, comme les filles ont la même liberté de choix que les garçons et font les mêmes gestes qu'eux, elles se retrouvent sur un certain pied d'égalité avec eux. Toutefois, cette égalité n'existe que parce que l'enjeu du couronnement s'avère peu important. En effet, il n’implique aucune décision d'ordre économique ou politique, deux domaines dans lesquels la liberté d'action, qui est synonyme d'un réel degré d'autonomie, est réservée aux hommes depuis les débuts de la colonie au XVIII ${ }^{\mathrm{e}}$ siècle ${ }^{42}$. Le couronnement à la fin

38. Anonyme, «Robert M. Evans papers», Rare Books and Manuscripts, Indiana State Library, <http:// www.in.gov/library/files/S440_Evans_Robert_M_Papers.pdf >.

39. Le journal de voyage que tient MacDonald pendant son séjour aux États-Unis a été publié par l'une des descendantes de Robert Owen, Caroline Dale Snedeker. Pour plus de détails, voir Donald MacDonald, "The Diaries of Donald Macdonald, 1824-1826», introduction de Caroline Dale Snedeker, Indiana Historical Society Publications, 14, 2 (1942), p. 151-158.

40. D. MacDonald, "The Diaries...», p. 272. Le baiser se fait sur la joue, et non sur la bouche, car cela constituerait une offense. En ce sens, les habitants suivent la tradition française du baiser social. En effet, selon Keith Thomas, aux XVIII ${ }^{\mathrm{e}}$ et XIX ${ }^{\mathrm{e}}$ siècles, "The English social kiss between men and women had been on the lips [...], whereas the French kiss on the cheeks». Pour plus de détails, voir Keith Thomas, "Afterword», dans Karen Harvey, dir., The Kiss in History (Manchester et New York, Manchester University Press, 2005), p. 194.

41. Martine Segalen affirme que les rituels de courtoisie qui laissent l'initiative aux jeunes filles existent également dans certaines régions françaises. Pour plus de détails, voir Martine Segalen, Amours et mariages de l'ancienne France (Paris, Bibliothèque Berger-Levrault, 1981), p. 65-67.

42. S. C. Boyle, «Did She Generally Decide?... », p. 775-789; W. E. Folley, The Genesis of Missouri..., p. 108-111. 
des bals permet donc d'observer une manifestation de l'égalité dans les rapports entre les genres dont la portée est somme toute secondaire et symbolique.

En fait, les bals des Rois mettent surtout en lumière l'existence concrète d'une hiérarchie entre les deux genres. Par exemple, leur organisation et leur préparation sont des tâches qui incombent uniquement aux quatre rois. Pour chaque bal, ils doivent payer les frais de la soirée. Ces tâches permettent aux garçons d'avoir un meilleur contrôle que les filles sur le marché matrimonial ${ }^{43}$. Ils doivent aussi sortir leur porte-monnaie pour offrir de beaux cadeaux à la reine qu'ils choisissent ${ }^{44}$. Le plus souvent, ce sont des vêtements que la reine exhibe fièrement lors du bal. Lors de son périple dans le Midwest américain, le New-Yorkais Christian Schultz s'arrête pendant plusieurs semaines à Sainte-Geneviève au cours de l'hiver 1808. Il a alors l'occasion d'assister à plusieurs bals des Rois ${ }^{45}$. Dans le récit de son voyage qu'il publie deux ans plus tard, il souligne que les quatre nouveaux rois ont la responsabilité de fournir à la fille qu'ils ont personnellement couronnée quelques objets qui font référence, de manière symbolique, aux apparats d'une reine, tels que des chaussures, des gants, des bas, des bracelets ou des boucles d'oreilles ${ }^{46}$. Robert Morgan Evans rapporte à Donald MacDonald que «it was customary to pay for a new ball-dress for his queen ${ }^{47}$ ». De son côté, sœur Philippine Duchesne, qui quitte la France en 1818, en compagnie de quatre consœurs de la congrégation du Sacré-Cœur de Jésus, pour venir fonder à Florissant un noviciat pour jeunes filles ${ }^{48}$, relate, dans une lettre datant de février 1819, qu’à Saint-Charles, les "filles sont folles de la danse; bal toutes les semaines: l'une est reine, parée aux frais de son roi, ouvre le bal, embrasse les assistants; les autres, qui n'ont souvent ni pain ni linge, ont au moins des robes de mousseline garnies, etc. ${ }^{49}$ ». Ces

43. P. Ward, Courtship, Love, and Marriage..., p. 68-69.

44. La responsabilité des rois de payer pour les frais d'organisation des bals et pour les cadeaux qu'ils offrent à la reine qu'ils ont choisie est mentionnée par plusieurs auteurs : Christian Schultz, John Reynolds, James Hall, Donald MacDonald et sœur Philippine Duchesne.

45. Pour plus de détails sur la biographie et le récit de voyage de Christian Schultz, voir Peter G. Beidler, "Christian Schultz's Travels: A New Source for Huckleberry Finn?», English Language Notes, 28 (décembre 1990), p. 52-53; Peter G. Beidler, "Schultz, Christian, Jr. », dans J. R. LeMaster et James D. Wilson, dir., The Mark Twain Encyclopedia (New York et Londres, Garland Publishing, 1993), p. 663-664.

46. C. Schultz, Travels on an Inland Voyage..., p. 61.

47. D. MacDonald, «The Diaries...», p. 272.

48. Pour plus de détails sur la biographie et l'œuvre de sœur Philippine Duchesne, voir l'introduction de Chantal Paisant dans Philippine Duchesne et ses compagnes, Les années pionnières, 1818-1823: Lettres et Journaux des premières missionnaires du Sacré-Cœur aux États-Unis (Paris, Les Éditions du Cerf, 2001), p. 9-44.

49. Philippine Duchesne, «L 56 Philippine Duchesne à Madeleine-Sophie Barat, Saint-Charles, Missouri Territory, ce 15 février 1819 », dans P. Duchesne et ses compagnes, Les années pionnières..., p. 232. 
cadeaux possèdent une forte connotation symbolique, car ils «servent de truchement pour encourager le garçon à poursuivre ses avances». Les rois ont la responsabilité d'offrir et les reines ont le droit de refuser ou d'accepter l'avance. En portant habituellement fièrement les cadeaux vestimentaires offerts, elles acquiescent au renouvellement de la rencontre du prétendant sous la surveillance de la communauté et permettent à la relation de se développer ${ }^{50}$.

En ce qui concerne le montant total dépensé par les rois, Robert Morgan Evans débourse plus de $60 \$$ à Vincennes, tandis que Christian Schultz affirme ceci pour Sainte-Geneviève: "These balls are tolerably expensive for a town in the wilderness, as they generally cost from a hundred to a hundred and fifty dollars each ${ }^{51}$.» Sachant que le salaire annuel d'un engagé varie entre 140 et 160 \$ par année à la fin du XVIII siècle et que les meilleurs ouvriers de Saint-Louis font 3 \$ par jour en 1817, les sommes déboursées pour les bals semblent donc être assez élevées ${ }^{52}$. Cela montre que ces réjouissances sont pour eux des événements festifs et communautaires très importants. La préservation des institutions qui soudent leurs communautés est un enjeu de premier ordre. De plus, le fait que les coûts associés aux bals soient toujours à la charge des quatre rois et jamais à celle des quatre reines montre très bien que ces festivités sont des occasions de réaffirmer les codes sociaux de l'époque aux Illinois. En d'autres mots, les bals des Rois mettent en lumière que les jeunes filles ne sont toujours pas les égales des garçons, car lors de rituels de constitution des couples, elles sont déjà confinées dans un rôle de dépendance.

Les bals illustrent également que la hiérarchie sociale est un élément clé dans la manière de gérer les fréquentations entre les garçons et les filles. L'historiographie des Illinois est avare de détails sur les fréquentations entre les garçons et les filles avant le mariage, mais Carl J. Ekberg, Denise-Marie Wilson, Bonnie Stepenoff et Soazig Villerbu expliquent très bien que, tant au XVIII ${ }^{\mathrm{e}}$ qu'au XIX ${ }^{\mathrm{e}}$ siècle, les membres de la petite élite bourgeoise des villages comme Sainte-Geneviève et Vincennes ont tendance le plus souvent à se marier entre $e^{5} x^{53}$. Pour ces derniers, le mariage sert avant tout à créer des alliances entre les familles prééminentes, car ils veulent faire avancer

50. M. Segalen, Amours et mariages..., p. 59-63.

51. D. MacDonald, "The Diaries...», p. 272; C. Schultz, Travels on an Inland Voyage..., p. 62.

52. C. J. Ekberg, Colonial Ste. Genevieve..., p. 143-163, 476; W. E. Folley, The Genesis of Missouri..., p. 253.

53. C. J. Ekberg, Colonial Ste. Genevieve..., p. 179-181; D.-M. Wilson, «Vincennes... », p. 336-338; B. Stepenoff, From French Community to Missouri Town..., p. 140-141; Tangi Villerbu, "Amérique française et "microhistoire globale" : la famille Rozier dans le Kentucky et le Missouri, 1806-1860", Francophonies d'Amérique, 40-41 (automne 2015, printemps 2016), p. 122-125. 
leurs intérêts économiques en préservant la concentration de la richesse ${ }^{54}$. En fait, le mariage, dans les sociétés rurales préindustrielles, est presque toujours une transaction économique entre deux familles de même statut social qui veulent préserver leurs patrimoines ${ }^{55}$. Cette tendance explique pourquoi les jeunes célibataires épousent souvent des gens qui font partie de leur entourage immédiat ou de réseaux de connaissances ${ }^{56}$. Dans les villages des Illinois, au-dessous de l'élite bourgeoise, les différences entre les niveaux de la hiérarchie sociale de la communauté blanche francophone sont peu marquées. Il existe certes une minorité de très petits propriétaires fonciers, d'engagés et de journaliers qui possèdent peu de biens, mais au cours du XVIII ${ }^{\mathrm{e}}$ et au début du XIX ${ }^{\mathrm{e}}$ siècle, la majorité des habitants sont des propriétaires fonciers moyens entre qui les écarts de richesse sont peu élevés ${ }^{57}$. Les bals et le marché matrimonial que ces derniers incarnent concernent surtout ce groupe social.

Comme dans d'autres sociétés rurales à la même époque, les fréquentations dans les bals des Rois sont encadrées par la famille et la collectivité, et plus précisément par les aînés ${ }^{58}$. Ces derniers s'assurent que les jeunes respectent, lorsqu'ils choisissent la personne qu'ils fréquentent, la hiérarchie sociale prévalant dans la société des Illinois. En fait, ce rôle montre que la sociabilité est régie par des dynamiques de pouvoir. Les filles et les garçons peuvent aborder et couronner la personne qu'ils désirent, mais ils ne peuvent ensuite agir de n'importe quelle manière avec cette personne, car leurs gestes sont attentivement surveillés par les aînés afin qu’ils respectent un décorum. Par exemple, à Kaskaskia et à Cahokia, John Reynolds raconte que si la joie est au rendez-vous sur la piste de danse, le respect des bonnes manières doit être maintenu. Aucun alcool, cigare ou remarque déplacée n’est accepté. Les personnes les plus âgées du village

54. C. J. Ekberg, Colonial Ste. Genevieve..., p. 188-190; W. E. Folley, The Genesis of Missouri..., p. $105-106$.

55. P. Bourdieu, "Célibat...», p. 33-34; M. Segalen, Amours et mariages..., p. 16; P. Ward, Courtship, Love, and Marriage..., p. 140.

56. P. Ward, Courtship, Love, and Marriage..., p. 65-66; Martine Tremblay, Le mariage dans la vallée du Haut-Richelieu au XXe siècle: ritualité et distinction sociale (Sainte-Foy, Les Presses de l’Université Laval, 2001), p. 58-59.

57. C. J. Ekberg, Colonial Ste. Genevieve..., p. 181-184; W. E. Folley, The Genesis of Missouri..., p. 105-106; C. Vidal, "Africains et Européens au Pays des Illinois...», p. 58; R. M. Morrissey, "Bottomlands and Borderlands...", p. 281-288.

58. P. Bourdieu, «Célibat...», p. 33-34; M. Segalen, Amours et mariages..., p. 34-35 ; P. Ward, Courtship, Love, and Marriage..., p. 65-68; M. Tremblay, Le mariage..., p. 57-59, 66-69; Anne-Marie Desdouits, "Les rituels du mariage paysan sur la Côte-de-Beaupré et dans la Beauce», dans Gérard Bouchard et Serge Courville, dir., La construction d'une culture: le Québec et l'Amérique française (Sainte-Foy, Les Presses de l’Université Laval, 1993), p. 310-311. 
s'assurent du bon déroulement des soirées de bal: «The old people regulate all; the time to retire and the time to meet again. » Deux d'entre elles sont choisies pour être les prévôts. Leur tâche est de désigner les filles et les garçons qui vont danser, "so that each one dances in proper turn ${ }^{59}$ ", bien que Reynolds ne précise pas ce qu'il entend par un ordre approprié. De leur côté, les femmes surveillent d'un oil attentif les danseurs et les danseuses à l'esprit plus frivole afin de percevoir de possibles gestes qu'elles jugent déplacés. Il n'est pas rare non plus que «the priest attended the early part of the evening in the balls, and saw that the innocent and proper observance of just principles be the order of the party ${ }^{60}$ ». En 1823, la consœur de Philippine Duchesne, Lucile Mathevon, prétend que plusieurs mères amènent leurs filles, des novices du couvent de Florissant, aux bals du carnaval et «les forcent d'y aller. Lorsqu'une mère dit à sa fille: "Tu es une imbécile, vois si ta sœur fait tant de grimaces que toi, elle a plus d'esprit que toi», ces pauvres enfants se laissent entraîner ${ }^{61}$. " En affirmant cela, elle montre que les mères encadrent de près la participation de leurs filles. Selon Henry Marie Brackenridge, les aînés agissent ainsi, car ils cherchent à enseigner aux plus jeunes « a decorum and propriety of behavior, which is preserved through life ${ }^{62} »$. Cette surveillance et cet enseignement semblent efficaces. Brackenridge lui-même admire la politesse et le respect des participants aux bals, tandis que dans la première nouvelle de James Hall, le narrateur insiste sur leur gentillesse, leur aisance et leur élégance ${ }^{63}$. Ces exemples montrent que les jeunes des communautés blanches francophones peuvent se côtoyer, qu'ils soient bourgeois, enfants de modestes cultivateurs, engagés ou journaliers, mais lorsque vient le temps de danser avec une personne de l'autre sexe, ils doivent le faire dans le respect du cadre des conventions sociales de l'époque. Par leurs comportements, ils se soumettent et acceptent de facto le contrôle des aînés. En d'autres mots, lors de ces bals, les aînés encadrent un marché matrimonial dans lequel ils s'assurent que les jeunes choisissent de fréquenter une personne qui est du même statut social qu'eux. Ils cherchent ainsi à garantir un phéno-

59. J. Reynolds, The Pioneer History of Illinois..., p. 72.

60. John Reynolds, My Own Times : Embracing Also the History of My Life (Chicago, Chicago Historical Society, 1879), p. 38.

61. L'italique est utilisé dans le document original. Lucile Mathevon, «L 157 Lucile Mathevon à Madeleine-Sophie Barat, Florissant, 10 mars 1823 ", dans P. Duchesne et ses compagnes, Les années pionnières..., p. 557.

62. H. M. Brackenridge, Views of Louisiana..., p. 137.

63. J. Hall, «The French Village», p. 117. 
mène observé par l'historiographie au XVIII ${ }^{\mathrm{e}}$ siècle, c'est-à-dire la reproduction de l'ordre social des communautés blanches ${ }^{64}$.

La manifestation de la hiérarchie raciale dans le déroulement des bals des Rois constitue un autre aspect de l'analyse de la sociabilité de ces festivités. Les Autochtones et les Noirs, esclaves ou libres, sont totalement absents des célébrations, car comme au siècle précédent, les communautés conçues par les colons blancs francophones se limitent à eux seuls ${ }^{65}$. Les autres groupes de la société des Illinois ne sont pas présents dans les descriptions de leur univers festif, car la couleur de leur peau les en exclut. Ils sont considérés comme des subalternes ${ }^{66}$. Les Américains blancs ne sont en revanche pas écartés. Certains d'entre eux sont même invités à prendre part aux bals et y sont traités avec respect et gentillesse. Par exemple, Robert Morgan Evans est choisi comme roi par l'une des reines lors d'un bal à Vincennes ${ }^{67}$. De son côté, le narrateur de la première nouvelle de James Hall se fait dire que les étrangers y sont toujours les bienvenus. Il reçoit d'ailleurs un bel accueil:

The moment I entered the room, I felt that I was welcome. Not a single look of surprise, not a glance of more than ordinary attention, denoted me as a stranger or an unexpected guest. The gentlemen nearest the door bowed as they opened a passage for

64. L'historiographie ne précise pas si l'endogamie est répandue parmi la population blanche francophone des villages des Illinois dans les premières décennies du XIX ${ }^{\mathrm{e}}$ siècle. En l'absence de telles données, il est impossible de déterminer si le contrôle du marché matrimonial exercé par les aînés dans les bals des Rois permet de préserver entièrement la stabilité des familles comme au siècle précédent. Toutefois, l'analyse de ces bals montre que la reproduction sociale des familles blanches est une valeur très importante pour eux.

65. Les autorités civiles et religieuses interdisent le côtoiement entre Blancs et Noirs dans l'univers social. Différentes lois sont promulguées à cet effet au XVIII ${ }^{\mathrm{e}}$ siècle et dans la première moitié du XIX ${ }^{\mathrm{e}}$ siècle. Pour plus de détails, voir C. J. Ekberg, Colonial Ste. Genevieve..., p. 207-208, 233-234; W. E. Folley, The Genesis of Missouri..., p. 254; B. Stepenoff, From French Community to Missouri Town..., p. 49-54, 97-98.

66. Plusieurs historiens de la Louisiane des XVIII et XIX ${ }^{\mathrm{e}}$ siècles ont aussi montré que les maitres blancs ne se mélangent pas à leurs esclaves dans l'univers festif. Les membres de la population servile noire festoient habituellement entre eux. Pour plus de détails, voir A. P. Malone, Sweet Chariot..., p. 225-226; Gwendolyn Midlo Hall, Africans in Colonial Louisiana: The Development of Afro-Creole Culture in the Eighteenth Century (Baton Rouge, Louisiana State University Press, 1992), p. 320-331; Thomas N. Ingersoll, Mammon and Manon in Early New Orleans: The First Slave Society in the Deep South, 1718-1819 (Knoxville, University of Tennessee Press, 1999), p. 292-293, 305-306; Richard J. Follet, The Sugar Masters: Planters and Slaves in Louisiana's Cane World, 1820-1860 (Baton Rouge, Louisiana State University Press, 2005), p. 162-172, 213, 219-226. Il est légitime de croire que la population noire des Illinois organise également ses propres réjouissances populaires. En effet, en 1796, le curé de Sainte-Geneviève, Paul de Saint-Pierre, se plaint à l'évêque de la NouvelleOrléans, Luis Peñalver y Cárdenas, que des jeunes hommes libres ont la «fâcheuse» tendance d'organiser des bals le dimanche soir au cours desquels ils dansent avec des femmes noires, esclaves et affranchies comprises. Ces hommes libres sont probablement des «libres de couleur», c'est-à-dire des esclaves noirs affranchis. Pour plus de détails, voir Archives de l'Université Notre-Dame, Archidiocèse de la NouvelleOrléans, CANO IV-5-1, lettre de Paul de Saint-Pierre à M ${ }^{\mathrm{gr}}$ Luis Peñalver y Cárdenas, 30 septembre 1796.

67. D. MacDonald, «The Diaries...», p. 272-273. 
me through the crowd, in which for a time I mingled, apparently unnoticed. At length a young gentleman, adorned with a large nosegay, approached me, invited me to join the dancers, and, after enquiring my name, introduced me to several females, among whom I had no difficulty in selecting a graceful partner. I was passionately fond of dancing, so that, readily imbibing the joyous spirit of those around me, I advanced rapidly in their estimation ${ }^{68}$.

Cet exemple et celui d'Evans ne représentent toutefois pas une norme, car les Américains blancs ne semblent pas se retrouver fréquemment dans les bals et la plupart du temps, ils s'y présentent seuls. Cela s'explique par le fait que dans les premières décennies du XIX ${ }^{\mathrm{e}}$ siècle, les gens de condition modeste des deux groupes (francophones et anglophones) n'ont pas encore l'habitude de se côtoyer très souvent et de socialiser ${ }^{69}$. Malgré tout, l'acceptation de certains Américains dans les bals des Rois et le bel accueil qui leur est réservé mettent très bien en lumière la différence entre le traitement accordé à des personnes blanches et à celles d'origine autochtone ou africaine. En vertu de la couleur de leur peau, les Blancs peuvent être des candidats acceptables au marché matrimonial, mais pas ceux des deux autres groupes. En ce début du XIX ${ }^{\mathrm{e}}$ siècle, bien qu'une certaine historiographie semble idéaliser les francophones du Pays des Illinois en montrant qu'ils ont peut-être généralement de meilleurs rapports avec les membres des Premières Nations et avec leurs esclaves noirs que leurs homologues américains, il ressort qu'ils font preuve de la même conception de la hiérarchie raciale que ces derniers dans leur gestion de l'univers festif des bals des Rois $^{70}$.

\section{CONCLUSION}

L'analyse de l'univers festif des bals des Rois des habitants blancs francophones du Pays des Illinois permet de combler deux vides historiographiques. Premièrement, elle fait ressortir les mœurs de cette population dans les trois premières décennies du XIX ${ }^{\mathrm{e}}$ siècle. Deuxièmement, elle met en lumière les fonctions sociales de ces réjouissances populaires. Malgré les changements politiques, économiques et judiciaires qui ont cours au Pays des Illinois avec l'arrivée de plus en plus importante de colons américains,

68. J. Hall, «The French Village», p. 117.

69. D.-M. Wilson, «Vincennes... », p. 343-344.

70. Le concept biologique de la race et de la hiérarchie raciale qui prévaut dans les colonies anglophones d'Amérique du Nord a été largement étudié dans l'historiographie. Pour plus de détails à ce sujet, voir Alden T. Vaughan, Roots of American Racism: Essays on the Colonial Experience (New York, Oxford University Press, 1995) et le numéro spécial «Constructing Race», The William and Mary Quarterly, 54, 1 (janvier 1997), p. $1-252$. 
l'étude des bals des Rois montre que certains traits sociaux caractéristiques de la population blanche francophone de la région observés au XVIII ${ }^{e}$ siècle, tels que l'esprit communautaire et de cohésion, l'importance de la stabilité de la famille et les hiérarchies sociales et raciales, sont toujours perceptibles au début du siècle suivant, car ils sont à l'origine des singularités et des généralités de ces festivités.

Les bals des Rois sont également le théâtre de fréquentations entre les jeunes célibataires qui mettent en scène une sociabilité qui semble à première vue égalitaire. Cependant, il n'en est rien, car cette sociabilité met surtout en lumière la reproduction des hiérarchies de genre, de classe et de race qui existent dans cette société. Tout d'abord, l'égalité entre les genres est plutôt futile, car les filles sont le plus souvent assignées à un rôle de dépendance financière à l'égard des garçons. De plus, bien que l'esprit de cohésion permette le rassemblement de divers niveaux de fortune, les aînés des communautés s'assurent que les fréquentations se fassent seulement entre jeunes au statut social similaire. Ils cherchent ainsi à contrôler en quelque sorte le marché matrimonial dans l'objectif de garantir la stabilité des familles blanches francophones. Finalement, à l'instar de ce qui prévaut dans les autres sociétés esclavagistes de l'époque, le cadre festif des bals comprend exclusivement des villageois blancs, car les personnes d'origine autochtone ou africaine sont considérées comme des êtres inférieurs qui n’y ont pas leur place. Ces réjouissances populaires, intimement liées les unes aux autres, servent donc surtout à exprimer et à renforcer la solidarité entre Blancs francophones et à définir de manière implicite les balises qui caractérisent leur identité. 